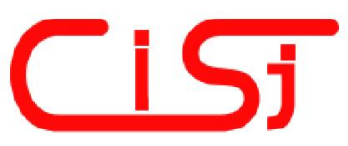

\title{
AN IMAGE-PROCESSING TECHNIQUE FOR GLAUCOMA DETECTION ON THE BASIS OF OPHTHALMIC IMAGES
}

\author{
Maroua Ben Mansour ${ }^{1)}$, Yosra Mlouhi ${ }^{2)}$, Imed Jabri ${ }^{1)}$, Tahar Battikh ${ }^{1)}$, \\ Lotfi Maalej ${ }^{1)}$, Mohamed Najeh Lakhoua ${ }^{3)}$
}

1) National Superior School of Engineering, University of Tunis, L.R: LATICE, Tunisia e-mail: mlouhi_yosra@yahoo.fr,imedjabri@yahoo.com, btahar@yahoo.com

2) Time university, 45 Avenue Mohamed V - Montplaisir 1002, Tunisia, mlouhi_yosra@yahoo.fr

3) National School of Engineering of Carthage, University of Carthage, U.R: SMS, Tunisia, e-mail: Inajeh@yahoo.fr

\begin{abstract}
In this paper, a new strategy of observation and analysis of the ophthalmologic images in order to detect the glaucoma is presented. In fact, we have treated the imagery applied to the Retina and we were able to develop some algorithm of digital image treatment, and the motif analysis techniques for the detection of the optic disc. And to do this, we have exploited the pretreatment of the image techniques such as morphological filters to improve the quality of the image and the Canny filter for the edge detection. Under the framework of the detection of circular shapes, we have opted the use of Algorithm which is the most famous detector of shapes and Hough Transform to detect the Papilla and Excavation. All these images will be applied on the fundus in order to create software illustrating some techniques of the image treatment, capable of determining precisely the relationship between the excavation diameter and the Papilla diameter which is a determining factor in the diagnosis of the Glaucoma. Copyright (C) Research Institute for Intelligent Computer Systems, 2015. All rights reserved.
\end{abstract}

Keywords: Ophthalmologic images analysis; Glaucoma; Papilla; Techniques of image pretreatment; Canny filter; Hough Transform; Excavation.

\section{INTRODUCTION}

This paper describes an application allowing the analysis, assisted by a computer, of medical images and more precisely ophthalmologic images to detect a chronic optic neuropathy named "Glaucoma" which is characterized by a slow and gradual destruction of the fiber layer of the optic nerve and which causes an irreversible blindness in the absence of adequate treatment [1].

The emergence of an optic nerve in the eye corresponds to the anatomic structure which we call "Papilla" or the optic disc "Disc", it's also the ocular anatomic structure which is destroyed gradually by the "Glaucoma" [2-5].

This alteration is distinguished by the appearance of an Excavation "Cup" or by the enlargement of a constitutional physiological excavation.

The result of the developed solution is the report Cup/Disc (Normally in the order of 0.3.(2)) which is evaluated horizontally and/or vertically.

It seems more relevant if we want to retain only one value, to consider the Cup/Disc vertically as in the Glaucoma, the Papilla excavates more vertically than horizontally. An augmentation of the report
Cup/Disc translates the thinning of the neuroretinal ring so an augmentation of the excavation size.

The literature course shows that, there are different works, treating the ophthalmologic images analysis but very few are designed to detect the Glaucoma such as the software GLAUCAD, certainly this software is payable, developed with "Matlab" and it is a closed group.

The goal of our work is to enforce a developed application with VBnet, which uses the functions predefined but the library OpenCv via the EmguCv [6] to treat the imagery applied to the retina, developing some techniques of image treatment for the ophthalmology.

In particular, we use an algorithm of digital images treatment and analysis techniques of the motif for the detection of the optic Papilla. And to do that, we have exploited some techniques of images treatment such as the morphological filters to improve the quality of the image and the filters detection of the edge for the pretreatment of the fundus images $[7,8]$, the Hough Transform for the detection of the circles.

All these methods will be applied on fundus images from a database provided by a team of 
Tunisian Ophthalmologists in order to localize the evolution of the excavation and to limit the optic Papilla and to perform quantative evaluation results of the detection of the optic Papilla with the help of different automatic measures.

\section{PROPOSAL OF A SOLUTION FOR GLAUCOMA DETECTION}

The proposed solution enables the ophthalmologist to choose the fundus image to be treated after doing his authentication.

Knowing that an image is specified by its genetic settings: color and depth to create a color image nonsigned of 8 bits in the EmguCV, the image is loaded in a picture box, visualized by the ophthalmologist (Fig. 1).

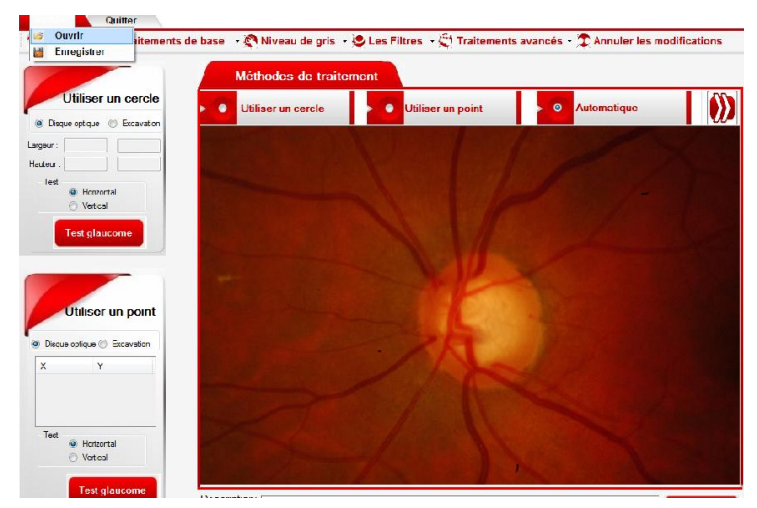

Fig. 1 - Fundus Image.

The approach of the proposed solution (Fig. 2) is essentially made of 3 steps:

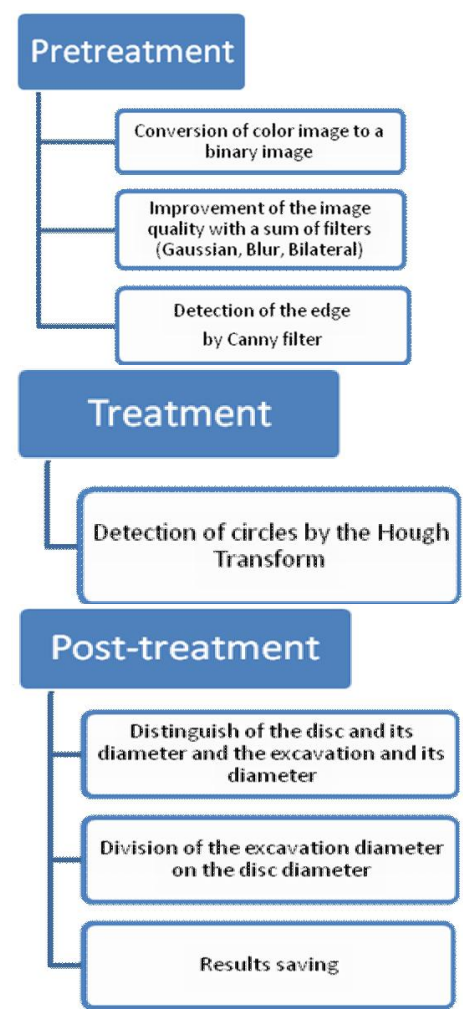

Fig. 2 - Structure of the proposed solution.
- Identification and localization of important anatomic structures from the treated image, this step is determinant in the process of images pretreatment.

- Images treatment by given information from the first step to detect circular shapes (disc and excavation) by the Hough Transform [9-11] on the resulting image of edge detection by Canny filter [12-14].

- The post-treatment is a decisive step in the exploitation of the obtained results by Hough Transform to distinguish between the disc diameter and the excavation diameter.

\subsection{THE PRETREATMENT}

To improve the image quality and to prepare it to optimize the recognition of circular shapes, it's necessary to make a series of pretreatments that gathers all the techniques allowing to obtain a result without noise (4) knowing that the image quality is not necessarily the same for a computer or a human operator.

Thereby, for our solution, the starting data is a color image if the fundus and the result is binary image. To obtain the adequate measures, the module of the developed starting image treatment is composing to 3 steps:

- Detection of shapes in an image of a grey level.

- Filter application on starting images to optimize the recognition of shapes.

- Detection of the edge through the resulting image of the second sub-step.

The detection of important anatomic structures and shape corresponds to a conversion of a color image to a binary image using the InRange function of OpenCv which settings are:

- The minimum value of the filter fixed at 0 for the blue color and 175 for the green color, and the red color could be changed by the user.

- The maximum value of the filter fixed at $100 \mathrm{fir}$ the blue and green, color, and at 255 for the red color.

To obtain a noise that corrupts the information and to optimize circular shapes recognition, we have used 3 filters of OpenCv at the entry image:

- The Blur filter, adds a fuzzy effect to a display object and its content.

- The bilateral filter which is a linear filter helps to preserve the discontinuity and smoothes the similar photometry areas.

- The Gaussian filter which is a special isotropic filter with specific mathematical characteristics.

Edge detection is a preliminary step for many applications in image processing. The contours are in fact rich indexes for later interpretation of the image. The purpose of edge detection is to identify 
areas of a digital image corresponding to an abrupt change in light intensity. These changes in the image properties generally reflect significant events or changes in the properties of the world.

They include discontinuities in depth, in the orientation of a surface, the properties of a material and in the lighting of a scene.

The method of Canny research areas where the variation of the light intensity is greatest. Hence, the edges are detected where the gradient is the largest value.

The contours are detected by deleting all the edges that are not related at least in a highly visible contour (some outline). During this step, we add all the strongly defined contours at double thresholding to the final image. Then we go one by one through the low contours: If a faint outline is connected to a strong outline, it is added to the final image. If not, the low contour is deleted.

We used the Canny filter of OpenCV, a contour detection algorithm advanced in an image. This algorithm is parameterized by:

- A convolution nucleus size (default $=3$ ).

- Two thresholding parameters, which should be neither too low to avoid the inclusion of noise, not too high, to avoid non-consideration contours (60 and 150).

The result is a drawing with delicate contours, like "comic". Then we applied a contrast to thereby obtain a binary image containing black color outline and the rest white.

\subsection{HOUGH TRANSFORM}

The Hough transform is firstly considered as a standard tool used in computer vision to detect lines, circles and ellipses. On the other hand, it is considered the most famous algorithm for the detection of shapes compared to other conventional methods of pattern recognition: Bayesian, estimation, parametric linear classifiers, neural networks, etc. Hence, the general principle of this method is to establish a projection between the space of the image and a space of parameters representing a desired shape.

Knowing that the basic principle for the detection of circles is very simple: you draw the contours of the image and for all points belonging to the contours, draw in the accumulator a circle of radius $R$.

If one seeks detecting a circle of radius $\mathrm{R}$ then the point of the battery receiving the most votes will be the center of the desired circle.

This method has certain strengths such as the detection of a circle if partially hidden or even if the image is very noisy. In addition, it is fast and very robust if we know the radius of the desired circle.
Certainly, this treatment requires approximate knowledge about the radius of the desired circle.

These steps are based on the coordination of potential circles identified in the accumulator and the contours localized by applying the Canny filter. To do this, the transformed Hough was paired with the information that we have, about the details of potential centers.

Not to mention that the Hough transform is based on a principle of batteries, similar to the step of detecting, potential centers of the circles. For an unknown radius circle, the use of accumulators in three dimensions will be appropriate. The first two dimensions express the coordinates of the circle while the third connects these details to a radius $r$. If we want to find circles of radii 8,9 and 10 units, we will have to build a battery comprising three levels, each in two dimensions.

Once our Hough batteries are initialized from an image of Canny contours, we literally draw circles in one of the batteries by matching the center of the circle with the coordinates of the contour pixel for a given radius. To trace the most efficient possible circles in the Hough accumulator, the use of the Bresenham method was employed.

\subsection{POST-TREATMENT}

After the detection of two circular shapes and the disk excavation by the Hough transform, we passed to the distinction between those two by taking account of the radius and based on that, the radius of the disk is higher than the excavation.

Afterwards, we went to the division of the diameter of the excavation on the diameter of the optical disc (compared cup/disc) which is a key factor in determining glaucoma, knowing that if:

- The result is superior than 0.3 so the eye is pathological.

- The result is inferior than 0.3 then the eye is normal.

\section{RESULTS OF THE PROPOSED SOLUTION}

We tried to make several glaucoma tests from a dozen of fundus images of patient's eyes.

We tested our application on the image of the fundus of a first patient (Fig. 3).

The test result shows that the eye is pathological. Knowing that, the division of the diameter of the excavation (equal to 117) by the disc diameter (equal to 171) gives a higher value to 0.3 (equal to $0.69)$.

All the previous steps can be detailed in the section performed by a simple click on the "glaucoma test" button, and the result will be displayed. 


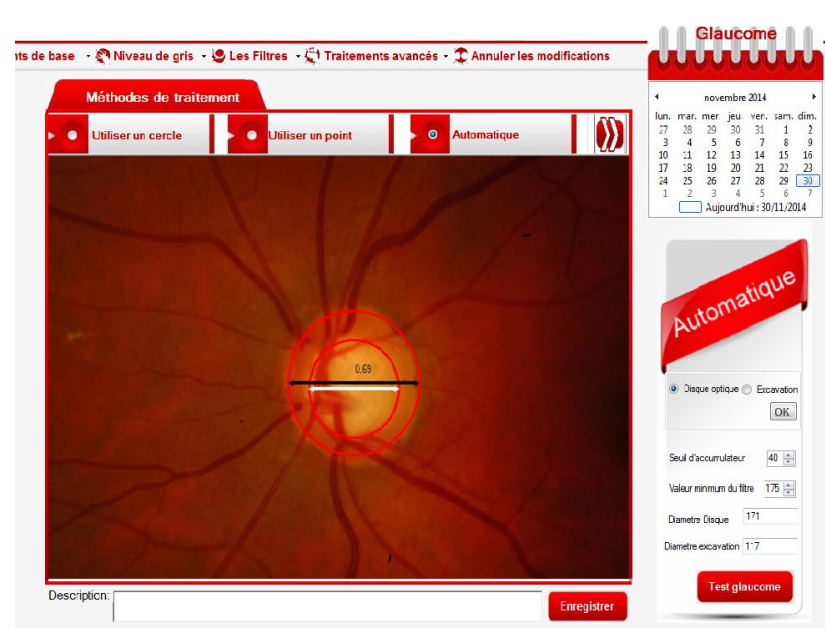

Fig. 3 - First result of the disk detection and excavation.

We tested our application on the image of the fundus of a second patient (Fig. 4).

The test result shows that the eye of the latter is pathological, since the division of the diameter of the excavation (equal to 110) by the diameter of the disc (equal to 182) which allows the have a value greater than 0.3 (equal to 0.61 ).
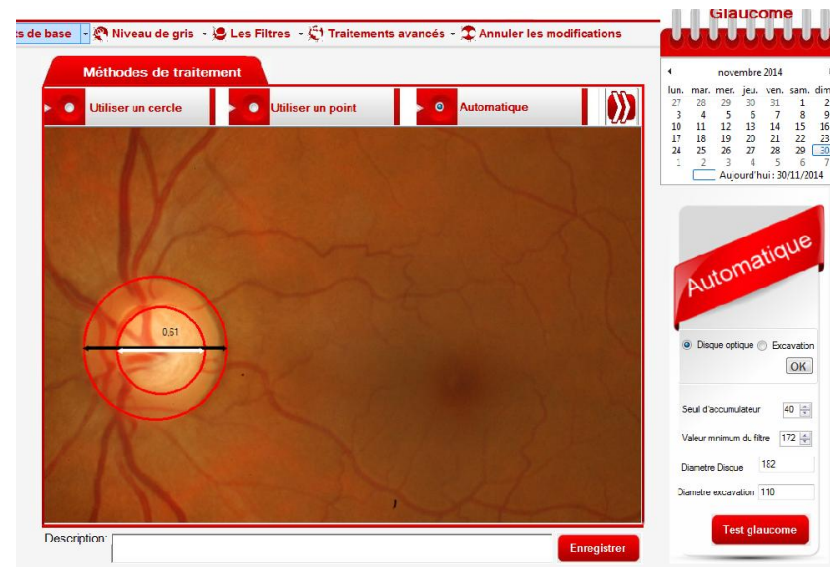

Fig. 4 - Second result of the disk detection and excavation.

We made a third test on another patient (Fig. 5), and we have determined that his eye is pathological by performing the division of the diameter of the excavation (equal to 116) by the diameter of the disc (equal to 161) and which has given a value superior than 0.3 (equal to 0.72 ).

Concerning the consultation of the evolution of the results over time, the ophthalmologist can save the results of the report cup / disc after each consultation in the database and can then see the evolution of this value in a patient especially over time by viewing the corresponding histogram (Fig. 6).

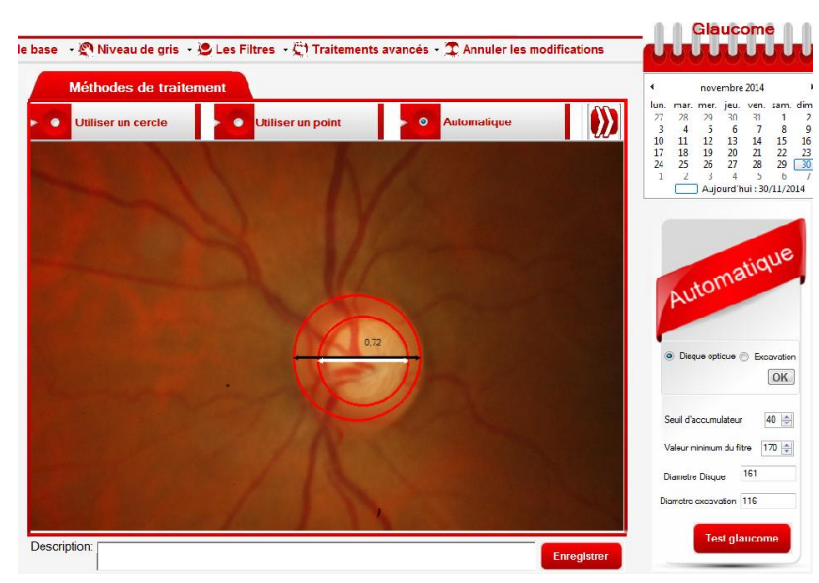

Fig. 5 - Third result of the disk detection and excavation.

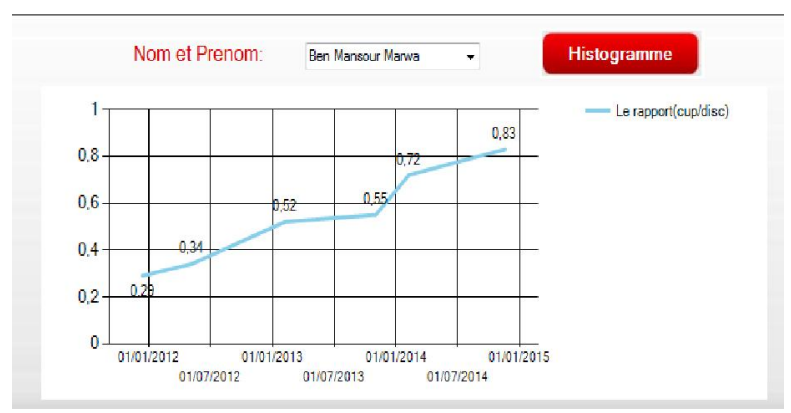

Fig. 6 - Histogram of the evolution of glaucoma.

In the previous part we presented some results of our application with the automatic method of the cup/disc in three patients report, and in this part we will make a comparison between the results of the automatic detection of the disk and excavation method and the results of the manual method.

To summarize, if the form of excavation is close to a circle, both methods will give the same result, but if the shape is close to an ellipse we will find a small difference because the Hough transform will detect a circular shape despite the fact that the shape can be distorted or stretched vertically or horizontally, circle where the usefulness of the manual method that takes into account any shape since it can make a horizontal test or even a vertical test.

\section{CONCLUSION}

In this paper, we have presented a solution which consists to develop excavation detection software of the optic nerve and which results in the detection of the disease of glaucoma, one of the principle causes of blindness which can fortunately be prevented through early detection. A set of software tools to optimize computer aided diagnosis of glaucoma for its early prevention was developed and we used real examples to illustrate and validate our approach. 
Table 1. Comparison between the results of the two methods (automatic and manual).

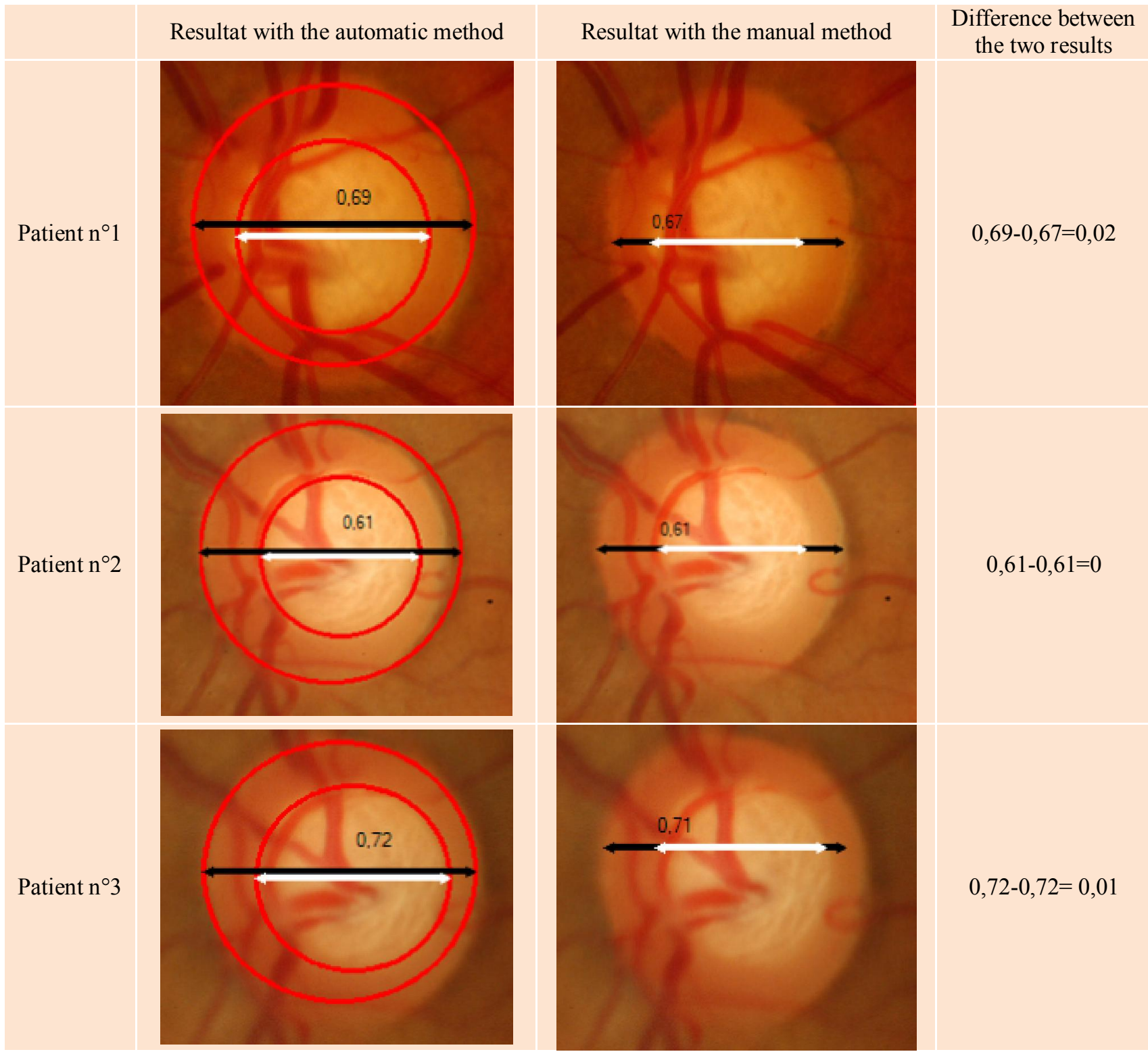

\section{REFERENCES}

[1] N. Dey, A.B. Roy, A. Das and S.S. Chaudhuri, Optical cup to disc ratio measurement for glaucoma diagnosis using harris corner, in Proceedings of the Third International Conference on Computing Communication \& Networking Technologies (ICCCNT), (26-28 July 2012), pp. 1-5.

[2] D. Lamani, T.C. Manjunath, A. Ramegowda, Fractal Dimension with Object Rotation: A Case Study with Glaucoma Eye, in Proceedings of the Fifth International Conference on Signal and Image Processing (ICSIP), (8-10 January 2014), pp. 111-116.

[3] U. Rajendra Acharya, S. Dua, D. Xian, S. Vinitha and C. Kuang, Automated Diagnosis of Glaucoma Using Texture and Higher Order Spectra Features, IEEE Transactions on
Information Technology in Biomedicine, (15) 3 (2011), pp. 449-455.

[4] C. Jun, L. Jiang, X. Yanwu and Y. Fengshou, Superpixel Classification Based Optic Disc and Optic Cup Segmentation for Glaucoma Screening, IEEE Transactions on Medical Imaging, (32) 6 (2013), pp. 1019-1032.

[5] L. Chow and M. Teymouri, Measurement of human optic nerve's diameter using magnetic resonance imaging (MRI) images, in Proceedings of the 5th International Conference on Intelligent and Advanced Systems (ICIAS), (3-5 June 2014), pp. 1-4.

[6] B.A. Wicaksana and R.F. Sari, Implementing text information display of detected color for partially color blinded person using .NET platform and EmguCV library, in Proceedings of the International Conference on Information Technology and Multimedia (ICIM), (14-16 
November 2011), pp. 1-6.

[7] A. Hani, T.A. Soomro, I. Faye, N. Kamel and N. Yahya, Denoising methods for retinal fundus images, in Proceedings of the 5th International Conference on Intelligent and Advanced Systems (ICIAS), (June 2014), pp. 1-6.

[8] V.K. Sree and P.S. Rao, Diagnosis of ophthalmologic disorders in retinal fundus images, in Proceedings of the Fifth International Conference on the Applications of Digital Information and Web Technologies (ICADIWT), (17-19 February 2014), pp. 131136.

[9] A. Bonci, G. Di Francesco and S. Longhi, A Bayesian approach to the Hough transform for video and ultrasonic data fusion in mobile robot navigation, in Proceedings of the IEEE International Conference on Systems, Man and Cybernetics, Vol. 3, (6-9 October 2002).

[10] O. Strauss, Reducing the precision/uncertainty duality in the Hough transform, in Proceedings of the International Conference on Image Processing, (16-19 September 1996), pp. 967-970.

[11] A.L. Kesidis and N. Papamarkos, On the inverse Hough transform, IEEE Transactions on Pattern Analysis and Machine Intelligence, (21) 12 (1999), pp. 1329-1343.

[12] L.H.A. Lourenco, D. Weingaertner and E. Todt, Efficient Implementation of Canny Edge Detection Filter for ITK Using CUDA, in Proceedings of the 13th Symposium on Computer Systems (WSCAD-SSC), (17-19 October 2012), pp. 33-40.

[13] Y. Huang, W. Weixing and L. WeiSheng, Anisotropic Filter Based Modified Canny Algorithm, in Proceedings of the Fourth International Conference on Fuzzy Systems and Knowledge Discovery (FSKD), Vol. 1, (24-27 August 2007), pp. 736-740.

[14] N. Begum, M. Alam and I. Islam, Application of Canny filter and DWT in fingerprint detection a new approach, in Proceedings of the 13th International Conference on Computer and Information Technology (ICCIT), (23-25 December 2010), pp. 256-260.

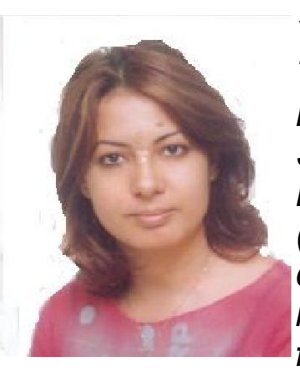

Yosra Mlouhi, born in 1977 in Tunis, Tunisia, received his Master's degree in Computer Science in 2003 from the Faculté des Sciences de Tunis (FST), she graduated from Specialized Master in Communication System and Network from the FST in 2005, she obta- ined a graduate degree in the specialty Communication System from the Ecole Nationale d'ingénieur de Tunis (ENIT) in 2008. In 2014, she's a PhD student at the Ecole Supérieure d'Ingénieurs de Tunis (ENSIT). Mme. Mlouhi Yosra has practiced as a student researcher at the FST from 2004 to 2008. Then as a contractual assistant at the Institut Supérieur d'Informatique de Tunis (ISI) from 2008 to 2013. Since 2013, she's assistant and researcher at Time University. His areas of interest are education research, Ambient Computing, Web Services, realtime systems, image processing to measure the technical and tactical performance of the game and players in sports, 3D real-time multimedia techniques in the field of sports and medical.

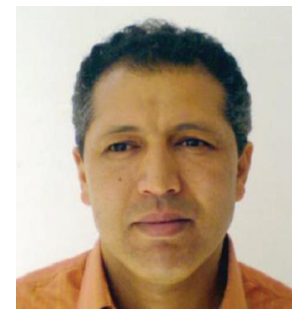

Imed Jabri, born in 1962 in Tunisia Ez-zahra, received his engineering degree in 1987 from the Institut National des Postes et Télécommunications de Rabat (INPT), specializing in audiovisual techniques, Rabat Morocco.

He obtained a Post Graduate Diploma in specialty Automatique de l'Ecole Normale Supérieure de l'Enseignement Technique ENSET (Tunis, Tunisia) in 1991. In 1996 he obtained his PhD from the Ecole Superieure des Sciences et Techniques de Tunis (ESSTT), Tunisia. From 1987 to 1993, he served as head of signaling and telecommunications service to the Société Nationale des Chemins de Fer Tunisiens (SNCFT). Since $1993 \mathrm{Mr}$ Imed JABRI occupies the position of researcher at ESSTT. His areas of interest are image processing to measure the technical and tactical performance of the game and players in sports, 3D real-time multimedia techniques and the use of GPS in the field of sport.

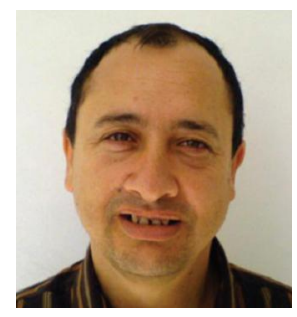

Tahar Battikh, born in 1964 in Tunisia Ez-zahra, receivedhis engineering degree in 1987 from the Ecole Supérieure d'Electricité Interafricaine (ESIE), Bingerville, Cote d'lvoire. In 1991, he received the Post Graduate Diploma in specialty Automatic Ecole Normale Supérieure de l'Enseignement Technique ENSET (Tunis, Tunisia). In 1996 he obtained his PhD from the Ecole Superieure des Sciences et Techniques de Tunis (ESSTT), Tunisia. $\mathrm{Mr}$ Tahar Battikh practiced as an engineer at the Société Tunisienne d'Electricité et du Gaz (STEG), Tunisia from 1987 to 1992, then as a researcher at the Institut National de Recherche Scientifique et Technique (INRST), Tunisia from 1992 to 1996. Since 1996 he is professor and researcher at the ESSTT. His research interests are in the field of image processing for observing and analyzing the performance of players and teams in sports, 3D real time, GPS technology and multimedia. 


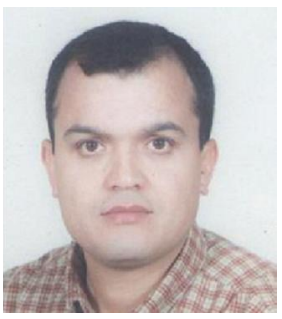

Lotfi Maalej, born in 1969 in Sfax, Tunisia, received his engeneering degree in 1992 from the Ecole Normale Supérieure de l'Enseignement Technique ENSET (Tunis, Tunisia), he obtained a Post Graduate Diploma in specialty

Automatique de l'Ecole Normale Supérieure de I'Enseignement Technique ENSET (Tunis, Tunisia) in 1995. In 2014, he obtained his PhD from the ENSIT, Tunisia. He served as trainer since 1992 at the Tunisian Agency for Vocational Training His areas of interest are the educational research, telemanipulation, image processing to measure the technical and tactical performance of the game and players in sports, 3D real-time multimedia techniques and the use of GPS in the field of sport.

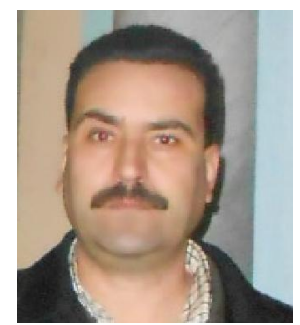

Mohamed Najeh Lakhoua, born in 1971 in Tunis (Tunisia), he received the $B S c$ degree in Electrical Engineering from the High School of Sciences and Techniques of Tunis, the DEA degree in Automatic and Production engineering from the same school and the PhD degree in Industrial Engineering from the National School of Engineers of Tunis, respectively in 1996, 1999 and 2008. He is currently an Assistant Professor at the National School of Engineering of Carthage (ENICarthage). In 2015, he obtained his HdR from the ENICarthage, Tunisia. $\mathrm{He}$ is a Senior Member of Institute of Electrical and Electronics Engineers (IEEE). Dr Lakhoua has published many scholarly research papers in many journals and international conferences. His research interests are focused on system modeling; automatic control; supervisory system; information system development. 